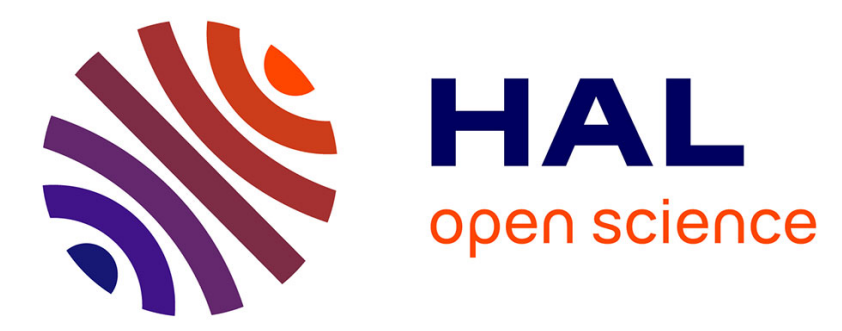

\title{
Réduction de la coarticulation et vieillissement
}

Daria d'Alessandro, Cécile Fougeron

\section{To cite this version:}

Daria d'Alessandro, Cécile Fougeron. Réduction de la coarticulation et vieillissement. XXXIIe Journées d'Études sur la Parole, 2018, Aix-en-provence, France. hal-02436286

\section{HAL Id: hal-02436286 \\ https://hal.science/hal-02436286}

Submitted on 5 Jan 2022

HAL is a multi-disciplinary open access archive for the deposit and dissemination of scientific research documents, whether they are published or not. The documents may come from teaching and research institutions in France or abroad, or from public or private research centers.
L'archive ouverte pluridisciplinaire HAL, est destinée au dépôt et à la diffusion de documents scientifiques de niveau recherche, publiés ou non, émanant des établissements d'enseignement et de recherche français ou étrangers, des laboratoires publics ou privés. 


\title{
Réduction de la coarticulation et vieillissement
}

\author{
Daria D'Alessandro \& Cécile Fougeron \\ Laboratoire de Phonétique et Phonologie - UMR 7018 \\ Université Sorbonne Nouvelle - CNRS \\ 19 Rue des Bernardins 75005 PARIS - France
}

daria.dalessandro@etud.sorbonne-nouvelle.fr,cecile.fougeron@univ-paris3.fr

\section{RESUME}

La parole évolue au cours de la vie avec le développement, chez les enfants, mais aussi avec le vieillissement, chez les adultes. Outre des modifications temporelles et spectrales connues, cette étude vise à examiner les effets du vieillissement sur la coarticulation anticipatoire voyelle_à_voyelle en français. Cet effet est testé en fonction de la durée des voyelles et de différences entre variantes régionales. Les productions de 167 locuteurs couvrant trois groupes d'âge (20-39) (50-69) (70-89) et 4 variantes régionales sont examinées. La coarticulation de V2 (/a/ ou /i/) sur V1 (/a/) est mesurée en termes d'abaissement de $F_{1}$ et montée de $F_{2}$. Les résultats montrent un degré de coarticulation et un allongement des voyelles variables en fonction de la variante régionale ainsi qu'un allongement global des voyelles chez les locuteurs les plus âgés. Plus intéressant, ces variations s'accompagnent d'une diminution de la coarticulation avec l'âge.

\section{ABSTRACT}

\section{Reduction in coarticulation and aging}

In the course of life, speech varies with respect to children's development but also with respect to aging. Besides the temporal and spectral modifications already attested, this study aims to investigate the effect of aging on vowel to vowel anticipatory coarticulation in French. This effect is tested according to vowel duration and to differences in regional varieties. Data from 167 speakers distributed across three age groups (20-39) (50-69) (70-79) and four regional varieties are investigated. The influence of $\mathrm{V} 2(/ \mathrm{a} /$ or $/ \mathrm{i} /)$ on $\mathrm{V} 1$ (/a/) is measured as a lowering of $\mathrm{F} 1$ and a rise of F2. Results show that coarticulation and vowel duration varies with regional variety and that vowels of older speakers are lengthened. More interestingly, results show a reduction of coarticulation with age.

MOTS-CLES : Coarticulation V à V ; vieillissement ; variété régionale du français KEYWORDS: $\mathrm{V}$ to $\mathrm{V}$ coarticulation; aging; French regional varieties 


\section{Introduction}

La coarticulation peut être définie comme le processus par lequel, dans la parole, un segment est modifié par les segments qui le précèdent (coarticulation persévérante) et/ou qui le suivent (coarticulation anticipatoire). Cette influence mutuelle entre les segments ne s'exerce pas seulement entre segments adjacents mais s'observe aussi à distance. La coarticulation a été beaucoup étudiée car elle soulève des questions relatives à l'organisation et la planification de la parole. En effet, si la coarticulation intra-syllabique et la coarticulation persévérante ont été attribuées, au moins en partie, à des contraintes biomécaniques, la coarticulation anticipatoire à distance a été vue comme la résultante de phénomènes anticipatoires lors de la planification de la parole (Whalen, 1990).

Un cas de coarticulation à distance est la coarticulation voyelle à voyelle $(\mathrm{V}-\mathrm{a}-\mathrm{V})$ : une voyelle exerce une influence sur une voyelle qui la suit ou qui la précède au travers des consonnes qui les séparent. Cette coarticulation $\mathrm{V}$-à-V dépend de différents facteurs, comme la nature de la (ou des) consonne entre la source et la cible. Ainsi, le degré de coarticulation V-à-V serait inversement proportionnel au degré de contact linguopalatal et d'implication du dos de la langue dans la séquence (Recasens, 1989 ; Mok, 2001). Une consonne palatale ou vélaire bloquerait d'avantage la coarticulation qu'une consonne bilabiale ; pareillement, les voyelles fermées /i/ et / $\mathrm{u} /$ seraient plus résistantes à la coarticulation que les voyelles ouvertes. Dans notre étude nous allons donc exploiter ces caractéristiques en nous focalisant sur des cas favorables à la coarticulation anticipatoire de $V_{2}$ sur $V_{1}$ dans des séquences $/ a p V_{2} /$.

Un facteur qui peut affecter la coarticulation est aussi l'âge du locuteur. L'effet de l'âge sur la coarticulation dans la parole a été, à notre connaissance, exclusivement focalisé sur la comparaison entre des groupes d'enfants et d'adultes d'âge moyen (et, dans une moindre mesure, entre adolescents et adultes), dans le cadre du développement de la parole. Les differentes études sur le sujet montrent globalement un changement dans les patrons de coarticulation entre enfants et adultes et une variabilité de la coarticulation plus forte chez les enfants par rapport aux adultes (inter alia Zharkova, 2012 ; Noiray, Ménard et Iskarous, 2013 ; Barbier et al., 2015). Toutefois, la direction du changement de la coarticulation n'est pas claire : certains montrent une coarticulation majorée chez les enfants par rapport aux adultes (Nittrouer et al., 1996; Zharkova et al,. 2011), d'autres montrent une coarticulation similaire (Rubertus et al., 2016) ou réduite (Zharkova, 2012 ; Barbier et al., 2016).

Si un effet de l'âge sur la coarticulation a été attesté en relation avec le développement du langage chez les enfants, les effets du vieillissement sur la coarticulation sont méconnus. Des effets du vieillissement sur la parole en général ont été documentés dans la littérature. Par exemple, il a été montré chez les personnes âgés un ralentissement de la parole (Jacewicz et al., 2009 ; BilodeauMercure et Tremblay, 2016), une augmentation de la variabilité de la durée des segments ou du VOT (Morris et Brown, 1984), des modifications de la $\mathrm{F}_{0}$ (diminution chez les femmes, augmentation chez les hommes, Torre et Barlow, 2009), une diminution de $F_{1}$, voire $F_{2}$ (Xue et Hao, 2003 ; Harrington et al., 2007). Ces effets du vieillissement sur la parole peuvent être liés à des changements dans les mouvements en général qui ont été largement documentés. En effet, les effets du vieillissement sur la 
cinématique des mouvements comprennent un allongement global de leur durée (de $30 \%$ à $60 \%$ en fonction des tâches motrices), une plus grande variabilité dans les trajectoires et les positions finales des mouvements, et une moins bonne coordination des mouvements (pour une revue, voir Ketcham et Stelmach, 2004). Par exemple Brown (1996) montre chez des adultes âgés (70-95) que la durée de simples mouvements du bras est plus variable, avec une asymétrie entre accélération et décélération du mouvement. Contreras-Vidal et al. (1998) observent une diminution de la coordination spatiale et une augmentation de la variabilité temporelle des mouvements avec le vieillissement. On peut donc se poser la question de savoir si ces changements peuvent affecter les temps et la coordination des gestes articulatoires dans la parole.

Connaitre l'évolution de la parole chez l'adulte est essentiel pour la recherche clinique, où il faut normaliser les données selon l'âge, mais aussi pour une meilleure compréhension générale du système de production de la parole. En effet, les changements liés à l'âge peuvent provenir soit de changements anatomiques et physiologiques dans l'appareil phonatoire, soit de changements neurologiques qui affectent le control moteur ou les fonctions cognitives (Torre et Barlow, 2009 ; Bilodeau-Mercure et al., 2016). C'est pourquoi dans cette étude, nous visons à examiner les effets possibles de l'âge des locuteurs sur la coarticulation anticipatoire V-à-V en français, en fonction aussi de l'origine du locuteur. En outre, nous allons examiner aussi les variations possibles liées à la dimension temporelle de la parole (ici des durées vocaliques) chez la personne âgée et la variabilité temporelle en fonction de la variété régionale, celle-ci pouvant aussi avoir un effet sur l'organisation temporelle de la parole (Jacevicz et al 2010, Verhoeven et al. 2004). Ces premières analyses des effets du vieillissement sur la coarticulation sont conçues comme préliminaires à des analyses sur un plus grand nombre de locuteurs.

\section{Méthode}

Un ensemble de 167 locuteurs, répartis sur 3 classes d'âges, 20-39, 50-69 et 70-89 ans, a été sélectionné dans la base de données MonPaGe_HA (Fougeron et al. 2018). Les caractéristiques des groupes d'âge sont présentées dans le tableau I. Les locuteurs (hommes et femmes) sont originaires de 4 régions francophones : région parisienne pour les locuteurs codés FR, région de Mons (Belgique) pour le code BE, région de Genève (Suisse) pour le code $\mathrm{CH}$, région de Montréal (Québec) pour le code QC. Ces locuteurs ont été enregistrés chez eux, par des proches ou des étudiants, selon un protocole dont la passation est informatisée et codifiée. Ce protocole d'évaluation de la parole, MonPaGe, cible diverses dimensions de parole pouvant être altérées en pathologie. Pour cette étude, la partie du protocole construite pour évaluer la production continue lors de la lecture d'un texte (188 mot, env. 1 min.) a été utilisée. Dans ce texte, 7 occurrences du mot 'Papa' et 5 occurrences du mot 'Papi' nous ont permis d'évaluer la coarticulation $V$-à- $V$ entre $V_{1}(/ a /)$ et $V_{2}(/ a /$ ou /i/) dans des séquences $/ \mathrm{pV}_{1} \mathrm{pV}_{2} /$.

Après segmentation manuelle, la durée des voyelles $\mathrm{V}_{1}$ a été mesurée, ainsi que la fréquence de ses $\mathrm{F} 1$ et $\mathrm{F} 2$. Comme l'effet coarticulatoire est légèrement plus fort sur la deuxième portion de la voyelle une moyenne des mesures à $60 \%, 70 \%$ et $80 \%$ de la durée de la voyelle pour F1 et F2 a été retenue. 
Une mesure composite de la compacité entre F1 et F2, représentée par la différence F2-F1 (toujours prise sur la partie $60-80 \%$ de la voyelle) a également été analysée mais ne sera pas présentée ici faute de place.

L'effet du type de $V_{2}(/ a /$ ou /i/) dont la significativité fera preuve de présence d'une influence coarticulatoire de $\mathrm{V}_{2}$ sur $\mathrm{V}_{1}$ ainsi que les interactions de ce facteur $\mathrm{V} 2$ avec l'âge des locuteurs (2039, 50-69, 70-89), leur origine (BE, CH, FR, QC), et leur sexe $(\mathrm{H}, \mathrm{F})$ sont testés à l'aide d'un modèle linéaire mixte construit à l'aide du package de R lme4 (Bates et al., 2015 ; R Core Team, 2017). Les variables dépendantes sont la durée et les $\mathrm{F} 1$ et $\mathrm{F} 2$ de $\mathrm{V}_{1}$. Les variations liées aux différences interlocuteurs sont modélisées par une structure aléatoire incluant des interceptes et pentes aléatoires par $\mathrm{V}_{2}$ par locuteur pour les formants, et un intercepte aléatoire pour la variable durée (la pente aléatoire n'améliorant pas le modèle dans ce cas). L'effet des facteurs fixes et de leur interaction est testé par comparaison de modèles avec la fonction anova. Les analyses des contrastes à postériori ont été effectuées avec la fonction lsmeans (bibliothèque 'emmeans', Lenth et al., 2018).

TABLE 1 : Effectif (N), Age moyen (en gras), écart type (entre parenthèse), âge minimum et maximum des locuteurs par groupes d'âge, sexe et variété régionale

\begin{tabular}{|c|c|c|c|c|c|}
\hline Groupe & Sexe & $\mathrm{BE}$ & $\mathrm{CH}$ & FR & QC \\
\hline $\begin{array}{c}\text { Gr1(20- } \\
39)\end{array}$ & $F$ & $\begin{array}{l}\mathrm{N}=8 ; 31(5), \\
23<>36\end{array}$ & $\begin{array}{l}\mathrm{N}=6 ; 27(4), \\
22<>35\end{array}$ & $\begin{array}{l}\mathrm{N}=8 ; 32(6), \\
23<>37\end{array}$ & $\begin{array}{l}\mathrm{N}=6 ; \mathbf{2 6}(4), \\
22<>33\end{array}$ \\
\hline & $\mathrm{H}$ & $\begin{array}{l}\mathrm{N}=8 ; \mathbf{3 0}(4), \\
25<>36\end{array}$ & $\begin{array}{l}\mathrm{N}=6 ; 27(5) \\
22<>36\end{array}$ & $\begin{array}{l}\mathrm{N}=8 ; \mathbf{2 9}(4), \\
24<>36\end{array}$ & $\begin{array}{l}\mathrm{N}=6 ; 28(5), \\
22<>35\end{array}$ \\
\hline $\begin{array}{c}\operatorname{Gr} 2(50 \\
69)\end{array}$ & $F$ & $\begin{array}{l}\mathrm{N}=8 ; \mathbf{5 9}(6), \\
50<>66\end{array}$ & $\begin{array}{l}\mathrm{N}=6 ; \mathbf{5 9}(7), \\
51<>69\end{array}$ & $\begin{array}{l}\mathrm{N}=8 ; \mathbf{5 9}(6), \\
51<>67\end{array}$ & $\begin{array}{l}N=6 ; 58(7), \\
50<>66\end{array}$ \\
\hline & $\mathrm{H}$ & $\begin{array}{l}\mathrm{N}=8 ; \mathbf{5 9}(6) \\
51<>67\end{array}$ & $\begin{array}{l}\mathrm{N}=5 ; \mathbf{5 9}(7), \\
51<>66\end{array}$ & $\begin{array}{l}\mathrm{N}=8 ; \mathbf{5 8}(7) \\
50<>68\end{array}$ & $\begin{array}{l}\mathrm{N}=6 ; \mathbf{5 9}(6), \\
50<>67\end{array}$ \\
\hline $\begin{array}{l}\text { Gr3(70- } \\
89)\end{array}$ & $F$ & $\begin{array}{l}\mathrm{N}=8 ; 79(7), \\
70<>89\end{array}$ & $\begin{array}{l}\mathrm{N}=6 ; 79(5), \\
73<>86\end{array}$ & $\begin{array}{l}\mathrm{N}=8 ; \mathbf{8 0}(4), \\
73<>85\end{array}$ & $\begin{array}{l}N=6 ; 78(7), \\
70<>86\end{array}$ \\
\hline & $\mathrm{H}$ & $\begin{array}{l}\mathrm{N}=8 ; \mathbf{8 0}(4), \\
75<>85\end{array}$ & $\begin{array}{l}\mathrm{N}=6 ; 79(8) \\
70<>88\end{array}$ & $\begin{array}{l}\mathrm{N}=8 ; \mathbf{8 0}(5), \\
72<>88\end{array}$ & $\begin{array}{l}N=6 ; 78(4), \\
71<>82\end{array}$ \\
\hline
\end{tabular}

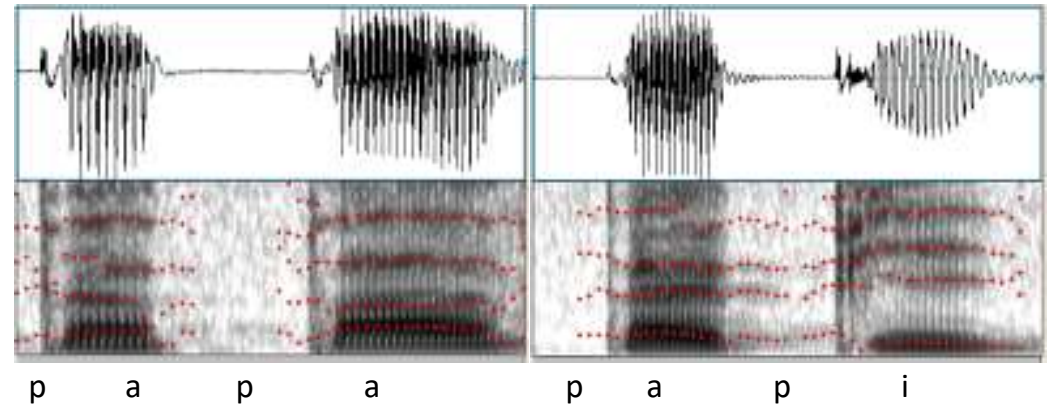


FIGURE 1 : illustration spectrographique de la variation de $\mathrm{V}_{1} / \mathrm{a} /$ en fonction de la voyelle suivante : /a/ dans 'papa' à gauche, /i/ dans 'papi' à droite, produits par la locutrice Locutrice FR_F_AB9, Gr1.

TABLE II. Résumé des effets fixes et interactions

\begin{tabular}{|l|l|l|l|}
\hline & F1 & F2 & Durée \\
\hline V2 & $\chi^{2}(1)=222,7 ; \mathrm{p}<.0001$ & $\chi^{2}(1)=222,7 ; \mathrm{p}<.0001$ & $\chi^{2}(1)=32,9 ; \mathrm{p}<.0001$ \\
\hline AGE & $\mathrm{ns}$ & $\chi^{2}(2)=15,9 ; \mathrm{p}<.0001$ & $\chi^{2}(2)=45,9 ; \mathrm{p}<.0001$ \\
\hline ORIGINE & $\mathrm{ns}$ & $\chi^{2}(3)=61,8 ; \mathrm{p}<.0001$ & $\chi^{2}(3)=25,4 ; \mathrm{p}<.0001$ \\
\hline SEXE & $\chi^{2}(1)=100,5 ; \mathrm{p}<.0001$ & $\chi^{2}(1)=98,2 ; \mathrm{p}<.0001$ & $\mathrm{~ns}$ \\
\hline V2 :AGE :ORIGINE & $\chi^{2}(12)=34,3 ; \mathrm{p}=.0006$ & $\chi^{2}(12)=31,1 ; \mathrm{p}=.0002$ & $\mathrm{~ns}$ \\
\hline V2 :AGE :SEXE & $\chi^{2}(4)=10,1 ; \mathrm{p}=.04$ & $\mathrm{~ns}$ & $\mathrm{~ns}$ \\
\hline V2 : AGE & $\chi^{2}(2)=16,1 ; \mathrm{p}=.0003$ & $\chi^{2}(2)=13,4 ; \mathrm{p}=.001$ & $\mathrm{~ns}$ \\
\hline V2 : ORIGINE & $\chi^{2}(3)=49,3 ; \mathrm{p}<.0001$ & $\chi^{2}(3)=63,8 ; \mathrm{p}<.0001$ & $\chi^{2}(3)=13,4, \mathrm{p}<.0001$ \\
\hline V2 : SEXE & $\chi^{2}(1)=6,6 ; \mathrm{p}=.009$ & $\mathrm{~ns}$ & $\mathrm{~ns}$ \\
\hline
\end{tabular}

\section{Résultats}

L'effet contextuel de $\mathrm{V}_{2} / \mathrm{i} /$ sur $\mathrm{V}_{1} / \mathrm{a}$ / se traduit par un abaissement de $\mathrm{F} 1$ et une élévation de $\mathrm{F} 2$ par rapport à la condition où $\mathrm{V}_{1}$ est suivi d'un $\mathrm{V}_{2} / \mathrm{a} /$, comme l'illustrent les exemples présentés Figure 1 . Combinés ces effets ont pour conséquence une diminution de la compacité F2-F1 en contexte V2 /i/. Les effets principaux obtenus sur la mesure de F1 et F2 de $\mathrm{V}_{1}$ sont donnés dans la Table II et illustrés dans la Figure 2. Un effet significatif sur $F 1$ est trouvé pour le facteur $\mathrm{V}_{2}$ avec une baisse significative de $\mathrm{F} 1$ en contexte $\mathrm{V}_{2}(\mathrm{i})(\mathrm{t}(167.8)=11.7 ; \mathrm{p}<.0001)$, et pour le facteur SEXE avec sans surprise des $\mathrm{F} 1$ globalement plus bas chez les hommes $(\mathrm{t}(174.3)=11.5$, $\mathrm{p}<.0001)$. Plus intéressant, nous observons une interaction significative entre l'effet de $V_{2}$ et tous les autres facteurs, et même une interaction triple. Ainsi, l'effet de $V_{2}$ dépend du groupe d'âge et de l'origine des locuteurs. Pour les locuteurs BE, il n'y a pas d'effet de $\mathrm{V}_{2}$ sur F1 (i.e. pas d'effet contextuel), ceci dans aucun des groupes d'âge. Par contre dans les trois autres variétés régionales, la baisse de $\mathrm{F} 1$ dans la condition $\mathrm{V}_{2} / \mathrm{i} /$ est significative, mais elle dépend des groupes d'âge : elle est significative pour les 3 groupes d'âge chez les QC $(\mathrm{Gr} 1(\mathrm{t}(182.6=5.4 \mathrm{p}<.0001), \mathrm{Gr} 2(\mathrm{t}(178.6)=6.5 \mathrm{p}<.0001), \mathrm{Gr} 3(\mathrm{t}(208.9)=5.2 \mathrm{p}<.0001)$ et les $\operatorname{FR}(\operatorname{Gr} 1(\mathrm{t}(176.3)=7,9 \mathrm{p}<.0001), \operatorname{Gr} 2(\mathrm{t}(183,7)=7,1 \mathrm{p}<.0001), \operatorname{Gr} 3(\mathrm{t}(185,9)=5.6 \mathrm{p}<.0001)$, mais la différence entre $\mathrm{V}_{2} / \mathrm{a}$ / et $\mathrm{V}_{2} / \mathrm{i} /$ diminue pour le groupe le plus âgé. Ceci apparait clairement sur la Figure 2, pour les femmes FR et QC, mais pas pour les hommes. Pour les locuteurs $\mathrm{CH}$, par contre, la baisse de $\mathrm{F} 1$ en $\mathrm{V}_{2} / \mathrm{i} / \mathrm{n}$ 'est significative que pour les locuteurs des deux premiers groupes $(\operatorname{Gr} 1(t(176.2)=8,8 \mathrm{p}<.0001), \operatorname{Gr} 2(\mathrm{t}(180,9)=3,4 \mathrm{p}<.009)$, alors que le groupe 3 (les plus âgés) se distingue par une absence d'effet contextuel, ce qui est clairement visible sur la Figure 2 pour les deux sexes.

Les effets de $\mathrm{V}_{2}$ sur le $\mathrm{F} 2$ de $\mathrm{V}_{1}$ sont illustrés sur la partie droite de la Figure 2. Un effet significatif est trouvé pour tous les facteurs fixes. En effet si le F2 de /a/ est globalement élevé par $\mathrm{V}_{2} / \mathrm{i} /$, il varie 
aussi selon l'âge, l'origine et le sexe du locuteur. Pour ce qui nous intéresse, nous observons que l'effet de $\mathrm{V}_{2}$ interagit avec l'âge et l'origine du locuteur : une augmentation significative de F2 en contexte $\mathrm{V}_{2} / \mathrm{i} /$ par rapport à $\mathrm{V}_{2} / \mathrm{a} /$ est trouvée pour tous les groupes d'âge et origines (et les deux sexes). Les interactions montrent que l'effet contextuel sur F2 (comme vu pour F1) varie en fonction des groupes d'âge, avec une augmentation dont le degré diminue globalement avec l'âge $(+248 \mathrm{~Hz}$ en moyenne pour le $\mathrm{Gr} 1$ et $+178 \mathrm{~Hz}$ pour le Gr3), mais d'avantage dans certaines variétés régionales que d'autres. Ainsi, comme illustré sur la figure 2, la différence entre $\mathrm{V}_{2} / \mathrm{i} /$ et $\mathrm{V}_{2} / \mathrm{a}$ / tend à diminuer chez les $\mathrm{CH}$ et FR (pour les deux sexes) et les femmes QC entre le groupe le plus âgé comparé aux deux autres, alors que pour les BE un effet d'âge est moins clair.

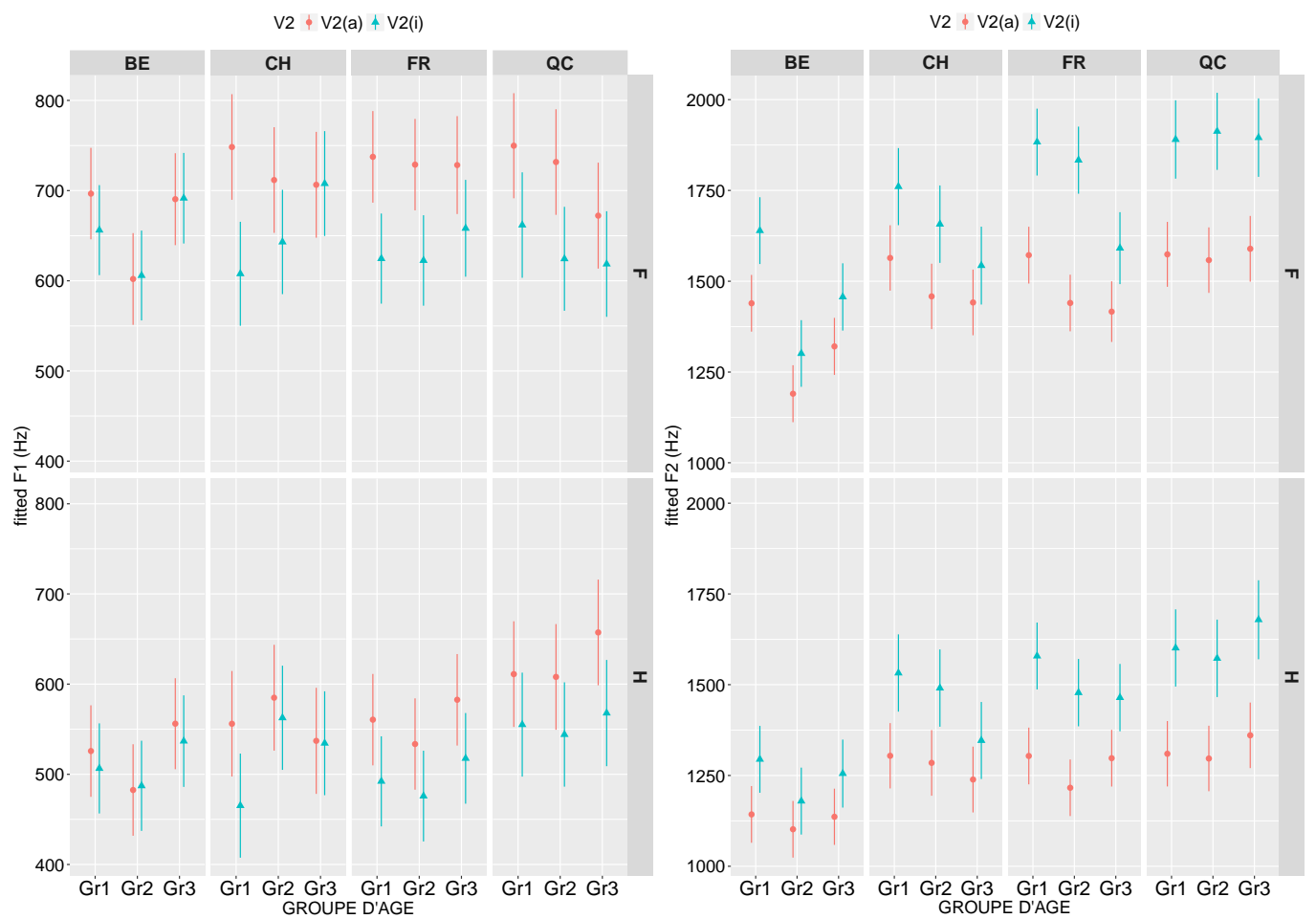

FIGURE 2 : Effets de V2 sur F1 (à gauche) et F2 (à droite) de V1 en fonction du groupe d'âge, de l'origine du locuteur et du sexe.

Enfin, concernant la durée de la voyelle $V_{1}$, un effet significatif est trouvé pour tous les facteurs fixes à part le $\mathrm{SEXE}$, ainsi qu'une interaction $\mathrm{V}_{2}$ :ORIGINE. Les voyelles $\mathrm{V}_{1}$ sont globalement plus longues lorsqu'elles sont suivies d'une $\mathrm{V}_{2} / \mathrm{i} /$ que d'une $\mathrm{V}_{2} / \mathrm{a} /(\mathrm{t}(167,2)=5,3$, $\mathrm{p}<.0001)$, mais cet effet dépend de l'origine des locuteurs et ne s'avère significatif que chez les BE et les QC. D'autre part, la durée de $\mathrm{V}_{1}$ est globalement plus courte chez les locuteurs du premier groupe (20-39) que chez les plus âgés : 50-69 $(\mathrm{t}(74,8)=-7, \mathrm{p}<.0001)$, et 70-89 $(\mathrm{t}(175)=-4,8, \mathrm{p}<.0001)$ et chez les locuteurs BE et FR, 
que chez les $\mathrm{CH}$ et QC. Afin d'observer la relation entre degré de coarticulation et durée des voyelles, une différence $\left(\mathrm{V}_{2} / \mathrm{a}-\mathrm{i} /\right)$ a été calculée par locuteur entre la moyenne des $\mathrm{V}_{1}$ suivies de $\mathrm{V}_{2} / \mathrm{a} /$ et la moyenne des $\mathrm{V}_{1}$ suivies de $\mathrm{V}_{2} / \mathrm{i} /$ pour $\mathrm{F} 1$ et pour $\mathrm{F} 2$. Ces différences donnent une estimation de la coarticulation par locuteur et celles-ci ont été mises en relation avec la durée moyenne des voyelles du même locuteur (toute $\mathrm{V}_{2}$ confondues). La figure 3 illustre l'absence de corrélation globale entre ces deux dimensions $\left(\mathrm{r}^{2}=0,08\right.$ pour $\mathrm{F} 2$ et $\mathrm{r}^{2}=-0,04$ pour $\left.\mathrm{F} 1\right)$ : les locuteurs avec des voyelles les plus longues n'ont pas moins de différences entre les deux contextes (i.e. moins d'effet contextuel), même dans le groupe des locuteurs les plus âgés (Gr3).

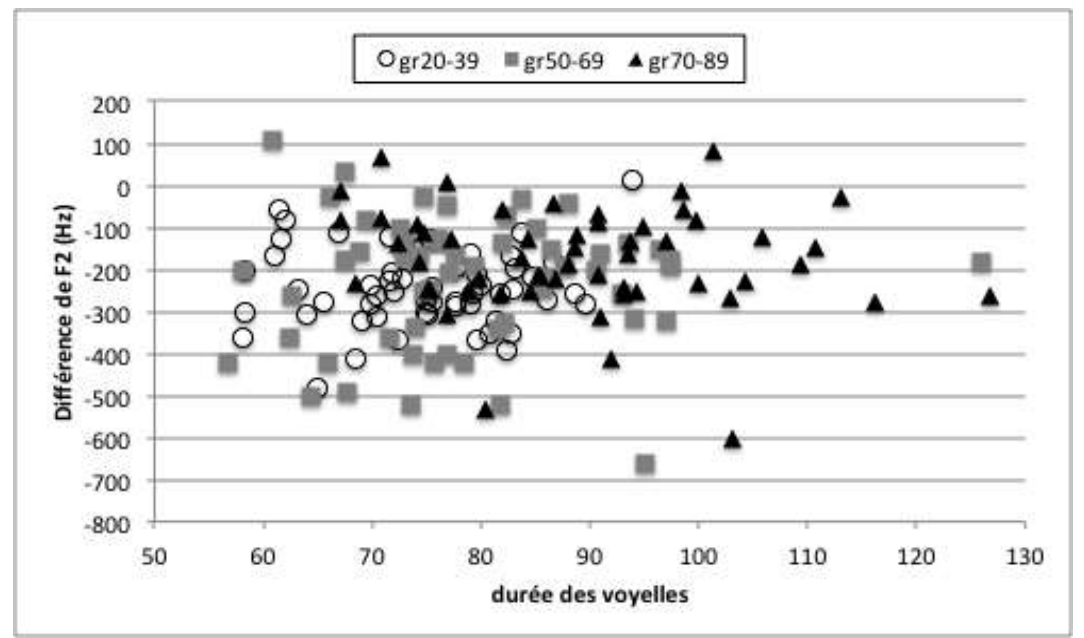

FIGURE 3 : Différence moyenne de $\mathrm{F} 2$ entre $\mathrm{V}_{2} / \mathrm{a} /$ et $\mathrm{V}_{2} / \mathrm{i} /$ par locuteur en fonction de la durée moyenne de ses voyelles (tous $\mathrm{V}_{2}$ confondues).

\section{Discussion}

Dans cette étude, nous avons examiné les productions de locuteurs de quatre variétés régionales de français et couvrant trois classes d'âge (20-39, 50-69, 70-89), pour tester si une variation dans la coarticulation $\mathrm{V}$-à-V peut être attribuée au vieillissement. Cette variation a été aussi examinée en rapport avec la durée de la voyelle et la variété régionale du locuteur.

Les résultats montrent une différence significative dans la réalisation de /a/ en fonction du contexte pour tous les groupes d'âge : l'anticipation d'un /i/ suivant abaisse le F1 et élève le F2 de /a/. Toutefois, malgré des différences régionales, on observe une diminution du degré de coarticulation avec l'âge, surtout chez les 70-89. Les locuteurs après 50 ans montrent aussi un allongement des voyelles. Les deux phénomènes de réduction de la coarticulation et d'allongement des voyelles chez les personnes âgées peuvent-ils être expliqués par les modifications observées sur d'autres 
mouvements avec l'âge? Si on considère la variation dans la coarticulation et dans la durée des voyelles comme le résultat de modification au niveau du control moteur, on pourrait lier ces phénomènes à un ralentissement des mouvements et à une altération de la coordination entre mouvements qui ont été associés au vieillissement. Moins de coarticulation et un ralentissement des mouvements vocaliques pourraient être attribués à une diminution de la précision en vitesse, qui a été avancée comme une des raisons du ralentissement des mouvements chez les adultes âgés : en se focalisant sur la production correcte d'une cible acoustique, il y aurait un ralentissement dans l'articulation des segments et une diminution de la coarticulation. Une autre explication serait que la moindre coarticulation traduirait une moins bonne coordination entre mouvements et donc un encodage plus séquentiel. Une variabilité majorée dans les mouvements a été aussi associé au vieillissement, et il est également possible qu'un accroissement de la variabilité générale des cibles vocaliques (non testée ici) noie la variabilité due au contexte. Enfin il est tout à fait possible que les capacités d'anticipation des mouvements à venir ou des unités linguistiques à venir changent aussi avec l'âge : cette possibilité a été explorée dans la littérature en relation aux changements dans la coarticulation de l'enfance à l'âge adulte. Ces différentes hypothèses devront être testées à l'avenir.

La coarticulation, et donc sa réduction avec l'âge, dépendent de la variété régionale. Un effet de l'âge est clairement visible chez les locuteurs parisiens et suisses et chez les femmes québécoises. Pour les locuteurs belges par contre, il n'y a pas d'effet contextuel sur F1 et peu d'effet sur F2. Cette moindre coarticulation et l'absence de diminution avec l'âge pourraient s'expliquer par le fait que dans ces groupes de locuteurs, notamment dans les groupes plus âgés, de nombreux locuteurs utilisent une variante postérieure, $[\mathrm{a}]$, avec un F2 bien plus bas que dans les autres régions. Une analyse plus poussée des 48 locuteurs BE est donc nécessaire. De la même façon, chez les québécois, on est en présence d'une variante beaucoup plus antérieure de type [æ] marquée par un F2 plus élevé. Outre des cibles de $V_{1}$ régionalement marquées, des différences dans le degré de coarticulation $\mathrm{V}$-à- $\mathrm{V}$ en fonction des variétés régionales apparaissent. Une différence d'influence contextuelle de $\mathrm{V}_{2}$ sur $\mathrm{V}_{1}$ en français a déjà été montrée dans des cas d'harmonie vocalique, i.e. un cas particulier de coarticulation V-à-V qui a été phonologisée en français. Nguyen et Fagyal (2008) ont montré que le degré d'harmonie vocalique diffère entre le nord et le sud de la France : si une telle assimilation régressive est présente chez tous les locuteurs dans une certaine mesure, celle-ci est plus grande chez les locuteurs parisiens que chez les locuteurs du sud. A notre connaissance, il n'existe pas d'autres études sur les effets de la variété régionale sur la coarticulation.

Enfin, les résultats montrent que la variété régionale affecte aussi la durée des voyelles. En effet, la durée de la voyelle est plus longue chez les locuteurs suisses et québécois, par rapport aux autres groupes. En outre, chez les locuteurs belges et québécois, la voyelle /a/ est significativement plus longue lorsqu'elle est suivie par un /i/ que par un /a/. Ces variations de durée selon le contexte pourraient être mises en relation avec les variations dans la coarticulation. En fait, les deux variétés régionales qui montrent le moins d'effet coarticulatoire, les belges et les québécois (pour les hommes) sont celles qui montrent un allongement de /a/ quand elle est suivie par/i/. Cet allongement pourrait contrecarrer l'assimilation de la voyelle, en permettant à la cible /a/ d'être atteinte. 


\section{Remerciements}

Ce travail est partiellement financé par le Labex EFL (ANR-10-LABX-0083) et le projet Sinergia du FNS MoSpeeDi. Nous remercions nos collègues du projet MonPaGe pour le partage des données et F. Ivent pour ses conseils statistiques.

\section{Références}

BARBIER, G., PERRIER, P., MÉNARD, L., PAYAN, Y., TIEDE, M., \& PERKELL, J. (2017). Speech motor control in 4-year-old children versus adults: anticipation as an index of speech motor control maturity. In 7th International Conference on Speech Motor Control.

BILODEAU-MERCURE, M., \& TREMBLAY, P. (2016). Age differences in sequential speech production: articulatory and physiological factors. Journal of the American Geriatrics Society, 64(11).

BROWN, S. H. (1996). Control of simple arm movements in the elderly. In Advances in psychology $114,27-52$

FOUGERON C., DELVAuX V., MÉNARD L., LAGANARO M. (2018) The MonPaGe_HA Database for the Documentation of Spoken French Throughout Adulthood, Actes de LREC 2018.

HARRINGTON, J., PALETHORPE, S., \& WATSON, C. I. (2007). Age-related changes in fundamental frequency and formants: a longitudinal study of four speakers. In Eighth Annual Conference of the International Speech Communication Association.

JACEWICZ, E., FOX, R. A., WEI, L. (2010) Between-speaker and within-speaker variation in speech tempo of American English. JASA 128(2), 839-850.

KETCHAM, C. J., \& STELMACH, G. E. (2004). Movement Control in the Older Adult. Technology For Adaptive Aging, 64.

MOK, P. P. (2011). Effects of vowel duration and vowel quality on vowel-to-vowel coarticulation. Language and speech, 54(4), 527-545.

MORRIS, R. J., \& BROWN JR, W. S. (1994). Age-related differences in speech variability among women. Journal of Communication Disorders, 27(1), 49-64.

NGUYEN, N., \& FAGYAL, Z. (2008). Acoustic aspects of vowel harmony in French. Journal of Phonetics, 36(1), 1-27.

NITTROUER, S., STUDDERT-KENNEDY, M., \& NEELY, S. T. (1996). How children learn to organize their speech gestures: Further evidence from fricative-vowel syllables. JSLHR, 39(2), 379-389.

NOIRAY, A., MÉNARD, L., \& ISKAROUS, K. (2013). The development of motor synergies in children: Ultrasound and acoustic measurements. JASA, 133(1): 444-52. 
R CORE TEAM (2017). R: A language and environment for statistical computing. R Foundation for Statistical Computing, Vienna, Austria. URL https://www.R-project.org/

RECASENS, D. (1989). Long range coarticulation effects for tongue dorsum contact in VCVCV sequences. Speech Communication, 8(4), 293-307.

SERENO, J. A., BAUM, S. R., MAREAN, G. C., \& LIEBERMAN, P. (1987). Acoustic analyses and perceptual data on anticipatory labial coarticulation in adults and children. JASA, 81(2), 512-519.

SMITH, B. L., WASOWICZ, J., \& PRESTON, J. (1987). Temporal characteristics of the speech of normal elderly adults. JSLHR, 30(4), 522-529.

TORRE III, P., \& BARLOW, J. A. (2009). Age-related changes in acoustic characteristics of adult speech. J Lang Commun Disord, 42(5), 324-333.

VERHOEVEN, J., DE PAUW, G., KLOOTS, H. (2004) Speech rate in a pluricentric language: A comparison between Dutch in Belgium and the Netherlands. Language \& speech, 2004, 47(3), $297-$ 308 .

CONTRERAS-VIDAL, J. L., TEULINGS, H. L., STELMACH, G. E. (1998). Elderly subjects are impaired in spatial coordination in fine motor control. Acta psychologica, , 100, 1-2, 25-35.

VOUSDEN, J. I., \& MAYLOR, E. A. (2006). Speech errors across the lifespan. Language and Cognitive Processes, 21(1-3), 48-77.

WHALEN, D. H. (1990). Coarticulation is largely planned. Journal of Phonetics, 18, 3-35.

XUE, S. A., \& HAO, G. J. (2003). Changes in the human vocal tract due to aging and the acoustic correlates of speech production: a pilot study. JSLHR 46(3), 689-701.

ZHARKOVA, N., HEWLETT, N., \& HARDCASTLE, W. J. (2011). Coarticulation as an indicator of speech motor control development in children: An ultrasound study. Motor Control, 15(1), 118140.

ZHARKOVA, N., HEWLETT, N., \& HARDCASTLE, W. J. (2012). An ultrasound study of lingual coarticulation in/s V/syllables produced by adults and typically developing children. JIPA 42(2), 193208. 